\title{
Planar Mechanism Incidence Matrices and Main Diagonal Spectrum ZHAO Kai
}

(Modern Manufacturing Engineering Department of Yibin Vocational and Technical College, Yibin, 644003, China)

EMAIL:kijidi@sina.com

Keywords: planar mechanism; incidence matrices; main diagonal spectrum;

\begin{abstract}
This paper applied the matrix theory and graph theory to the properties of planar mechanisms and constructed incidence matrixes. A construction method of characteristic matrices was proposed, and links incidence and pair incidence matrices were also defined. Meanwhile, through the concept of main diagonal spectrum, the physical meaning of the main diagonal spectrum was depicted in planar mechanism.
\end{abstract}

\section{Introduction}

Research on planar mechanisms involves structure, kinematics, and dynamics ${ }^{[1-3]}$, and matrix analysis and calculations are generally used as tools in mechanism studies. In recent years, scholars have made good progress on planar mechanism research. LI Shu-jun ${ }^{[4]}$, LIU Jiang-nan ${ }^{[5]}$, WU Yan-rong and JIN Guo-guang ${ }^{[6]}$, LU Wu-yun ${ }^{[7]}$, ZHANG Zhong-hai, and LI Duan-ling ${ }^{[8]}$ investigated and found several methods for rod adjacency matrices of planar mechanisms.

DAI Jian-sheng ${ }^{[9]}$ established a calculus matrix and topological structure in the planar mechanism method of matrix for the Assur group. LIU Chuan-he ${ }^{[10]}$ put forward the theory of variable topology structure in planar mechanisms. Yuan Qing-ke ${ }^{[11]}$ offered an incidence equation using graph theory, and studied the identification of kinematical chains, which led to useful results.

The focus of this paper is mainly on the structural attributes of planar mechanism, which were introduced into the matrix theory ${ }^{[12,13]}$ and graph theory ${ }^{[14-16]}$ to analyze the attribute structure of planar mechanisms. Due to their superior, Introducing the mechanism of matrix is an inevitable choice for modern science, and since the computation ability of a matrix is excellent, introducing it into the mechanism study was necessary. Mechanical matrix should contain the complete information of mechanism attributes.

Through matrix operation results, characteristics of various data can be depicted and characteristic mechanisms discovered. The application of computer-aided mechanical systems is getting more and more advanced, and the development of computation abilities has greatly helped the study of mechanisms.

\section{Definition of the incidence matrix of mechanical systems}

Definition 1: $G$ is the Planar mechanism system with $m$ kinematical pairs and $n$ links, the matrix $K(G)=\left(k_{i j}\right)_{m \times n}$ is defined as the incident matrix with linkages and pairs of planar mechanism $G, G=\langle P, L\rangle$, linkage set is $L=\left\{l_{1}, l_{2}, \cdots, l_{n}\right\}$, kinematical-pair set is $P=\left\{p_{1}, p_{2}, \cdots, p_{m}\right\} \quad, \quad l_{n}$ is the fixed link as last element for linkage set, $k_{i j}= \begin{cases}1, & \text { if } p_{i} \text { incident with } l_{j}, K(G) \text { also is called as the basic characteristics matrix of } \\ 0, & \text { if not }\end{cases}$ the mechanism $G$.

Definition 2: $P(G)$ is the kinematical-pair incidence matrix of planar mechanism $G, P(G)=K(G) K^{T}(G)$. The dimensions of $P(G)$ are $(m \times m)$ obviously and the kinematical-pair incidence matrix $P(G)$ is a symmetric matrix. 
As for matrix $P(G)$, the number on the diagonal of the matrix represents the number of revolute pairs between connected links. The digit 1represents the connection of links. Otherwise, the number will be 0 .

Theorem 1: For kinematical-pair incidence matrix $P(G)$, where $p_{i i}=\sum_{i=1}^{m} p_{i j}(i \neq j)$ and $p_{i i}=\sum_{j=1}^{m} p_{i j}(i \neq j)$

Definition 3: $L(G)$ is the link incidence matrix of planar mechanism $G$, $L(G)=L^{T}(G) K(G)$. The dimension of $L(G)$ is $(n \times n)$ and the links incidence matrix $L(G)$ is a symmetrical matrix.

Theorem 2: The number on the diagonal of the links incidence matrix $L(G)$ represents the link connection.Digit1 of the matrix element represents that the link and the corresponding link are connected. Otherwise, the value of the matrix element is 0 .

Theorem3: $L(G)$ is the link incidence matrix of the planar mechanism $G$, and if the diagonal of the matrix is set to be zero, $L(G)$ will become the adjacency matrix in the graph theory.

Definition 4: $\operatorname{Diag}-\operatorname{SpceP}(G)$ is defined as main diagonal spectrum of kinematical-pair incidence matrix of planar mechanism $G$, and $\operatorname{Diag}-\operatorname{Spce} L(G)$ is defined as main diagonal spectrum of the link incidence matrix of planar mechanism $G$, where main diagonal spectrum is the statistic for the values of the main diagonal elements in the incident matrix. .

$$
\operatorname{Diag}-\operatorname{SpceP}(G)=\left[\begin{array}{lllll}
\lambda_{1} & \lambda_{2} & \lambda_{3} & \ldots & \lambda_{s} \\
d_{1} & d_{2} & d_{3} & \ldots & d_{s}
\end{array}\right]
$$

where $\lambda_{i}$ is value in the incident matrix on the diagonal. For a matrix with a dimension of $m \times m$, it should have $n$ values. The same values of the diagonal in the matrix is denoted as $\lambda_{s}$, and the number of $i$ in the $\lambda_{i}$ is at most $m$, where $s=m$ is shown in the matrix that does not have the same value, and $m=\sum_{j=1}^{s} d_{j}$.

Due to the necessary movements of planar mechanisms, there should be a degree of freedom (DOF).Therefore, the structure of triangles of a mechanism system is rare, and both $L(G)$ and $P(G)$ are usually sparse matrices, which is beneficial for solving the determinant rank, which is very convenient for planar mechanism visualization of the matrix properties.

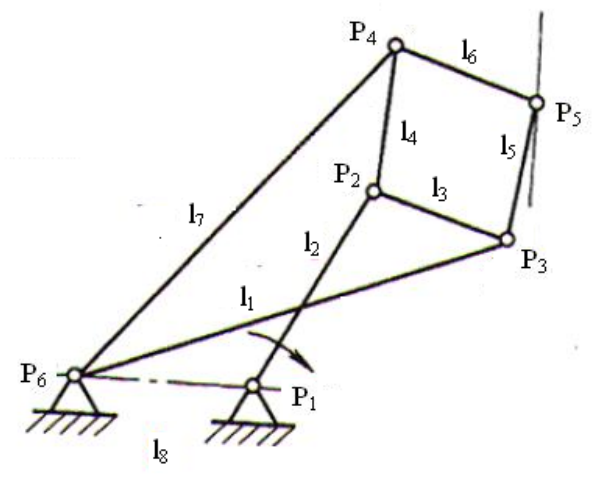

Fig. 1.Mechanism graph $G_{1}$ with 8 links and 6 kinematical pairs

From Figure 1, it can be seen that the incidence matrix $K\left(G_{1}\right), K^{T}\left(G_{1}\right), P\left(G_{1}\right)$ and $L\left(G_{1}\right)$ are: 


$$
\begin{aligned}
& \begin{array}{llllllll}
l_{1} & l_{2} & l_{3} & l_{4} & l_{5} & l_{6} & l_{7} & l_{8}
\end{array} \\
& K\left(G_{1}\right)=\left[\begin{array}{llllllll}
0 & 1 & 0 & 0 & 0 & 0 & 0 & 0 \\
0 & 1 & 1 & 1 & 0 & 0 & 0 & 0 \\
1 & 0 & 1 & 0 & 1 & 0 & 0 & 0 \\
0 & 0 & 0 & 1 & 0 & 1 & 1 & 0 \\
0 & 0 & 0 & 0 & 1 & 1 & 0 & 0 \\
1 & 0 & 0 & 0 & 0 & 0 & 1 & 1
\end{array}\right] p_{1} p_{2} p_{3} p_{p_{5}} \quad K^{T}\left(G_{1}\right)=\left[\begin{array}{llllll}
0 & 0 & 1 & 0 & 0 & 1 \\
1 & 1 & 0 & 0 & 0 & 0 \\
0 & 1 & 1 & 0 & 0 & 0 \\
0 & 1 & 0 & 1 & 0 & 0 \\
0 & 0 & 1 & 0 & 1 & 0 \\
0 & 0 & 0 & 1 & 1 & 0 \\
0 & 0 & 0 & 1 & 0 & 1 \\
0 & 0 & 0 & 0 & 0 & 1
\end{array}\right] \begin{array}{c}
l_{1} \\
l_{2} \\
l_{3} \\
l_{4} \\
l_{5} \\
l_{6} \\
l_{7} \\
l_{8}
\end{array}
\end{aligned}
$$

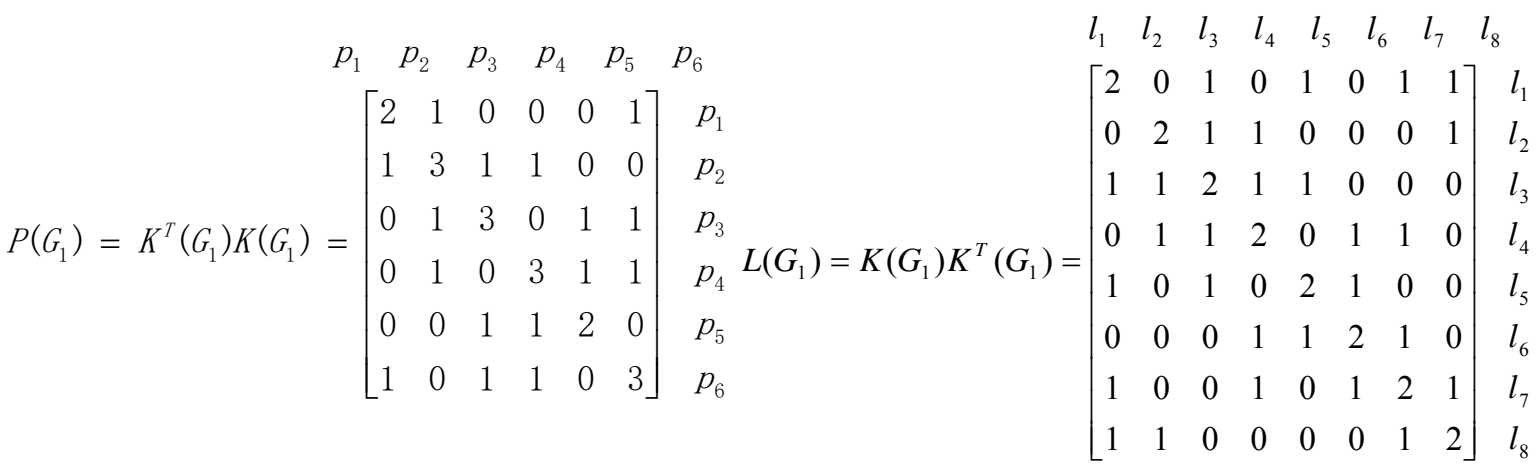

From the main diagonal elements of kinematical-pair incidence matrix $\operatorname{tr}(Z)=16$ can be seen, kinematical-pair incidence matrix trace, This mechanism has 2 single hinge and 4 2-complex hinge kinematical pairs, total pair numbers is 10 ; links incidence matrix trace is $\operatorname{tr}(L)=\operatorname{tr}(P)=16$, the mechanism have 8 links, each link has 2 kinematical pairs, $\operatorname{tr}(L)=\operatorname{tr}(P)=16$ can be obtained by matrix theory.

From the above calculation, $K(G), P(G), L(G)$ are relative matrices communications, and from anyone matrix of $K(G), P(G), L(G)$, another two matrices can be calculated. For general planar mechanisms, usually the number of kinematical pairs is less than the number of links, so the data of the kinematical-pair incidence matrix is less. The kinematical-pair incidence matrix is a symmetric matrix, from Theorem 1, and the unit of diagonal data of the kinematical-pair incidence matrix can be derived from the data of rows or columns of the adjacency matrix. Then, the actual stored data of the matrix on the computer is only $\frac{m^{2}-m}{2}$. Therefore, the kinematical-pair incidence matrix of planar mechanisms can contain as much with a less amount of data.

For the matrix $L\left(G_{1}\right)$ in Figure 1, it can be seen that the values of 2 for all links are provided with two pairs. The black box shown in the matrix $L\left(G_{1}\right)$ is the information vector for fixed link $(8)$. Links (1), (2), and (7) are connected to link (8), which is the fixed link.

In Figure (Example) 1, the diagonal spectrum of kinematical-pair incidence matrix of the mechanism can be obtained by:

$$
\operatorname{Diag}-\operatorname{SpceP}\left(G_{1}\right)=\left[\begin{array}{ll}
2 & 3 \\
2 & 4
\end{array}\right]
$$

The information of Diag-SpceP $(G)$ shows that there are four kinematical pairs that connect three links, and two kinematical pairs (A and E) that connect two links.

The diagonal spectrum of the links incidence matrix of the mechanism can also be provided in Example 1:

$$
\operatorname{Diag}-\operatorname{SpceL}\left(G_{1}\right)=\left[\begin{array}{l}
2 \\
8
\end{array}\right]
$$


The diagonal spectrum of the links incidence matrix indicates that the number of links of the two kinematical pairs for the mechanism is 8 , including a fixed link $1_{8}$.

\section{Formula for DOF of Mechanism}

In theory, although many controversies ${ }^{[17,18]}$ remain regarding the calculation of the DOF planar mechanism, the Grübler-Kutzbach formula is useful for calculating the degrees of freedom of mechanisms if they are not too complicated.. The formula $F=3 n-2 p_{L}-P_{H}$ has been widely used and approved by popular literature, and therefore, this paper also used it to calculate the DOF of the mechanism system. Since this paper only dealt with revolute pairs while the revolute pairs were the lower pair, the Grübler-Kutzbach formula could be simplified as $F=3 n-2 p_{L}$.

Through the pairs incidence matrix $P(G)$ or the links incidence matrix $L(G)$, estimating the formula for the degree of freedom of the mechanism system can be accomplished. The number of active links in the formula was $n-1$, that is, the fixed link was removed. The number of kinematical pairs was $\sum_{i=1}^{m} P(G)_{i i}-m$. So, the formula for the degree of freedom of the mechanism using the matrix $Z(G)$ mechanism system is:

$$
F=3 \times(n-1)-2 \times\left(\sum_{i=1}^{m} P(G)_{i i}-m\right)
$$

From Example 1, using Formula (2) can obtain the DOF of the mechanism $G_{1}$ :

$$
F=1
$$

\section{Conclusions}

(1) This paper put forward and defined the construction method of a basic characteristic matrix $K(G)$ for a mechanism system $G$, and also put forward and defined the link incidence matrix $L(G)=K(G) K^{T}(G)$, and the kinematical-pair incidence matrix $P(G)=K^{T}(G) K(G)$.

(2) $\operatorname{Diag}-\operatorname{SpceP}(G)$ and $\operatorname{Diag}-\operatorname{SpceL}(G)$ were put forward and defined in the matrix operation and their meanings for incidence matrix main diagonal spectrum of planar mechanism were explained.

(3) This paper is just studied and discussed for plane mechanism with all rotary pairs as an example, all incident matrixes have the vital significance to the study of rotary joints and moving pairs and spatial mechanism and metamorphic mechanism and its Mode conversion.

\section{References}

[1] HUANG Zhen, ZHAO Yong-sheng, ZHAO Tie-shi. Study of higher spatial mechanism [M].Beijing. Higher education press, 2006

[2] WAN Jing-ban。DANG Zhu-rang, SHENShou-fan . Application of Graph TheoryKinematical Chain in. Researchon mechanism kinematical chain isomorphism identificationusing adjacency matrix[J]. Chinese Journal of Mechanical Engineering。2004，40(7)

[3]ZOU Huijun, GAO Feng. Modem mechanism progress(Volume I)[M]. Beijing: Higher Education Press, 2007.

[4] LI Shu-jun, DAI Jian-sheng. Description of topological planar mechanism based on the Assur bar group elements[J]. Chinese Journal of mechanical engineering, 2011 (19)

[5] LIU Jiang-nan, YU De-jie. Description and analysis of spatial mechanism based on the constraint function of the adjacency matrix[J]. Chinese of mechanical engineering, 2012 (17) 
[6] WU Yan-rong, JINGuo-guang, LI Dong-fu et al. Adjacency matrix to describe the structure changing of metamorphic mechanisms[J]. Chinese Journal of mechanical engineering, 2007 (7)

[7] LU Wu-yun, LAN Zhao-hui. Improved adjacency matrix description of metamorphic mechanism configuration changes [J].. Mechanical transmission, 2009 (6)

[8] ZHANGZhong-hai, LIDuan-ling. Integrated structure of flexible metamorphic mechanism based on adjacency matrix operations[J]. Journal of BeijingUniversity of Posts and Telecommunications, 2009

[9] DAI Jian-sheng, DING Xi-lun, WANG De-lun. The topology of a spatial metamorphic mechanism and the corresponding matrix calculus $[\mathrm{J}]$. Chinese Journal of mechanical engineering, $2005(8)$

[10] LIUChuan-he, YANG Ting-li, LIU Yi. Basic problems of variable topology mechanism theory [J]. Chinese Journal of mechanical engineering, 2005 (8)

[11] YUAN Qing-ke, Li Wei-guang, LIU Da-hui et al. Application of technology in the identification of mechanical system graph algorithm of kinematic chain[J]. Journal of South China University of Technology, 2009 (2).

[12]. James E. Gentle. Matrix Algebra Theory, Computations, and Applications in Statistics [M] Springer Science \& Business Media,2007.

[13]. Alan Jeffrey. Matrix Operations for Engineers and Scientists [M] Springer Science \& Business Media B.V. 2010

[14]. J.A. Bondy, U.S.R. Murty. Graph Theory [M].Springer. 2008.

[15]. Handbook of Graph Theory (SECOND EDITION) [C]. CRC Press Taylor \& Francis Group.2014

[16] ZHANG Xian-di, LI Zheng-liang. Graph theory and its application [M]. Beijing. Higher education publishing, 2005, 15-20

[17] GUO Wei-dong, novel compliant linear guiding mechanism. A new method to calculate the degree of freedom of plane mechanism [J]. Chinese Journal of mechanical engineering, 2013 (07)

[18] HUANG Zhen, LIU Jing-fang, LIU Yan-wen. on the degrees of freedom of mechanism in [M]. Beijing: Science Press, 2011 